\title{
BMJ Open Effect of tobacco control policies on the Swedish smoking quitline using intervention time-series analysis
}

\author{
Xingwu Zhou, ${ }^{1}$ Alessio Crippa, ${ }^{2}$ Anna-Karin Danielsson, ${ }^{1}$ Maria R Galanti, ${ }^{1,3}$ \\ Nicola Orsini (D) ${ }^{1}$
}

To cite: Zhou X, Crippa A, Danielsson A-K, et al. Effect of tobacco control policies on the Swedish smoking quitline using intervention timeseries analysis. BMJ Open 2019;9:e033650. doi:10.1136/ bmjopen-2019-033650

- Prepublication history and additional material for this paper are available online. To view these files, please visit the journal online (http://dx.doi. org/10.1136/bmjopen-2019033650).

Received 15 August 2019 Revised 19 November 2019 Accepted 26 November 2019

Check for updates

(C) Author(s) (or their employer(s)) 2019. Re-use permitted under CC BY-NC. No commercial re-use. See rights and permissions. Published by BMJ.

${ }^{1}$ Department of Global Public Health, Karolinska Institutet, Stockholm, Sweden

${ }^{2}$ Department of Medical

Epidemiology and Biostatistics, Karolinska Institutet, Stockholm, Sweden

${ }^{3}$ Centre for Epidemiology and Community Medicine, Stockholm Health Care District, Stockholm County Council, Stockholm, Sweden

Correspondence to

Dr Nicola Orsini;

nicola.orsini@ki.se

\section{ABSTRACT}

Objectives To coherently examine the responsiveness of the Swedish National Tobacco Quitline (SNTQ) to different types of anti-smoking policies over an extended period of calendar time.

Design Quasi-experimental design with an intervention time-series analysis based on 19 years series of data collected between January 1999 and August 2017 (224 months). Statistical inference on calling rates and rate ratios was obtained using intervention time-series models (Poisson regression and transfer functions).

Participants A total of 179851 phone calls to the SNTQ. Interventions Recent application of the 2014/40/ European Union (EU) Tobacco Products Directive in 2016. Historical interventions such as a campaign on passive smoking in January 2001; introduction of larger text warnings on cigarette packages since September 2002; banning smoking in restaurants since June 2005; and tobacco tax increase by 10\% since January 2012.

Outcome measure Calling rates to the SNTQ expressed per 100000 smokers.

Setting Sweden.

Results The introduction of large pictorial warnings together with text warnings on cigarette packages (May 2016) was associated with a $35 \%$ increase in SNTQ calling rate $(95 \% \mathrm{Cl} 1.16$ to 1.57$)$. The campaign on passive smoking (Jan 2001) was associated with a $61 \%$ higher calling rate $(95 \% \mathrm{Cl} 1.06$ to 2.45$)$. Larger text warnings on cigarette packs (Sept 2002) conferred a $28 \%$ increment in the calling rate $(95 \% \mathrm{Cl} 1.15$ to 1.42$)$; and prohibition to smoke in restaurants (Jun 2005) was associated with a $15 \%$ increase in the calling rate $(95 \% \mathrm{Cl} 1.01$ to 1.30$)$. The $10 \%$ tobacco tax increase (Jan 2012) was associated with a $3 \%$ higher calling rate $(95 \% \mathrm{Cl} 0.90$ to 1.19$)$.

Conclusions Within an overall decreasing trend of daily smoking in Sweden, we found that the recent introduction of pictorial warnings together with text warnings and referral text had a discernible positive impact on the calling rates to the smoking quitline. We were also able to detect a likely impact of earlier nationwide interventions.

\section{INTRODUCTION}

Telephone smoking quitlines have the potential to reach a large proportion of the smoking population and therefore may play an important role in increasing the chances of smoking cessation. ${ }^{1}$ In addition, quitlines

\section{Strengths and limitations of this study}

To the best of our knowledge, this is the first consistent analysis of the effect of different types of tobacco control policies on the calling rates to a national tobacco quitline.

- The dynamic of the intervention effects over time has been modelled using advanced statistical methods yet using a small number of parameters.

- Multiple interventions are analysed using separate statistical models on definition of a pre/postintervention period.

- Possible sensitivity of the intervention effect to extreme values occurring right after intervention.

have been shown to be an effective ${ }^{2}$ and cost-effective modality for providing tobacco cessation interventions. ${ }^{3}$ To the best of our knowledge, however, no previous study has coherently examined the responsiveness of this service to different types of anti-smoking policies over an extended period of time.

The Swedish National Tobacco Quitline (SNTQ) is a nationwide service which started in 1998, with the intent to offer freeof-charge qualified in-person counselling to adult tobacco users, mainly smokers, wanting to quit. The service is operatively run by the Stockholm County Council, and is available during all week days for a total of 47 hours a week. The quit rates among a subsample of callers to the SNTQ have been estimated to be as high as $31 \%$ after 12 months from first approach. ${ }^{3}$ Since the very beginning the SNTQ has maintained a computerised system of registration of all incoming and outgoing calls, as well as selected information on individual callers who receive a counselling session.

The primary aim of this study is to investigate the possible effects of public health interventions on the volume of phone calls received by the SNTQ during the last 19 years, in particular the recent application of 
the 2014/40/ European Union (EU) Tobacco Products Directive, demanding member states of the European Union to insert larger pictorial warnings related to the health consequences of smoking on cigarette packs. On request of the Swedish Public Health Authority in Sweden, the application of the Directive also imposed a printed referral to the quitline, the toll-free number, which progressively appeared on all cigarette packages from May 2016. The secondary aim is to consistently evaluate tobacco control policies over the last two decades in which the Swedish government introduced campaigns on passive smoking in January 2001; larger text warnings on cigarette packs in September 2002; banning of smoking in restaurants in June 2005; and a $10 \%$ tax increase in January 2012.

\section{METHODS}

\section{Outcome data}

The number of calls to the smoking quitline was available from January 1999 to August 2017. A total of 179851 phone calls with requests of support to smoking cessation were received during 19 years, and aggregated into 224 months. To take into account the variation over calendar time of the number of smokers in the general adult population, we collected information on the total size of the Swedish population and smoking prevalence stratified by age groups and sex. The size of the population was available from Statistics Sweden on a yearly basis (http://www. scb.se). The smoking prevalence was updated every 2 years from the Swedish Living Conditions Surveys before 2004 and every year from the Public Health National Survey on tobacco use (http://www.folkhalsomyndigheten. se/) after 2004. The outcome of interest was defined as the calling rate expressed per 100000 smokers (ie, total number of phone calls divided by the total estimated number of smokers and then multiplied by 100,000 ).

\section{Tobacco control interventions}

Application of the 2014/40/EU Tobacco Products Directive

The latest implemented policy in Sweden, directly targeting smokers, concerns the application of the 2014/40/EU Tobacco Products Directive locally regulated by the Swedish government proposition 2015/16:82. According to these regulations starting from May 2016 the cigarette packages sold in Sweden had to display pictorial warnings together with text warnings. The coverage of all packages was completed in May 2017. In addition, the Swedish Public Health Agency negotiated that the packages should also display the phone number of the SNTQ together with a brief referral sentence.

We also identified other four main interventions aiming at reducing tobacco smoking in the population during the 19-year observation period: (1) a campaign on passive smoking in January 2001; (2) introduction of larger text warnings on cigarette packages since September 2002; (3) banning smoking in restaurants since June 2005; and (4) tobacco tax increase by 10\% since January 2012.
The calling rate during the month of January 2012 (297 per 100000 smokers) was fourfold higher than the average rate before the intervention (79 per 100000 smokers). The calling rate, however, dropped back to the pre-intervention level already in February 2012. This strong pulse on January 2012 at the start of the tobacco tax intervention was therefore excluded from the analysis to avoid overestimation of this specific intervention effect throughout the post-intervention period.

The unexpected surge in the number of calls in January 2012 may be an artefact due to registration in that month of calls incoming in the previous month of December 2011. Apart from a general tendency of higher number of calls in January across the years, we did not have the possibility to support this hypothesis with objective data. Therefore, this explanation remains speculative.

\section{Patient and public involvement statement \\ No patient involved.}

\section{Statistical analysis}

We used a Poisson regression model to estimate the linear trend of calling rates before and after the application of the 2014/40/EU Tobacco Products Directive. ${ }^{4}$ The model allowed a change in level (degree-0 spline) and trend (degree-1 or linear spline) after intervention. We evaluated the hypothesis of a change in the long-term trend after this intervention using a Wald-type test for the regression coefficient of the linear spline with one knot at May 2016. A scaling adjustment in Poisson regression was made to take into account possible over-dispersion (ie, conditional variance is higher than the conditional mean). We also adjusted for seasonal variation by modelling time with Fourier terms (three pairs of sine and cosine functions). Further details of this approach to interrupted time-series analysis have been described elsewhere. ${ }^{4}$

We next considered time-series models with transfer functions to disentangle the complex dynamic of the intervention effects from the noise of the series. ${ }^{56}$ These models are established in the economic and environmental literature but not so popular among health researchers probably because of its complexity, lack of specific statistical training and user-friendly software packages. The idea is to model pre-intervention rates with an autoregressive model to predict post-intervention rates in the absence of intervention, commonly defined as counterfactual rate. The intervention effect is given by the ratio of the observed rates divided the counterfactual rates, and this done for every month of the post-intervention period. The intervention effect is therefore varying over the post-intervention period. Different dimensions (form, reactivity, maintenance) of the intervention effect can be described with a transfer function involving only two parameters $\left(\omega_{0}, \delta\right)$. We choose the symbols $\omega_{0}$ and $\delta$ to be consistent with the notation commonly used for this class of dynamic models. The online supplementary appendix provides a more detailed description of the model, graphical intuition of the shape of the intervention effect and 


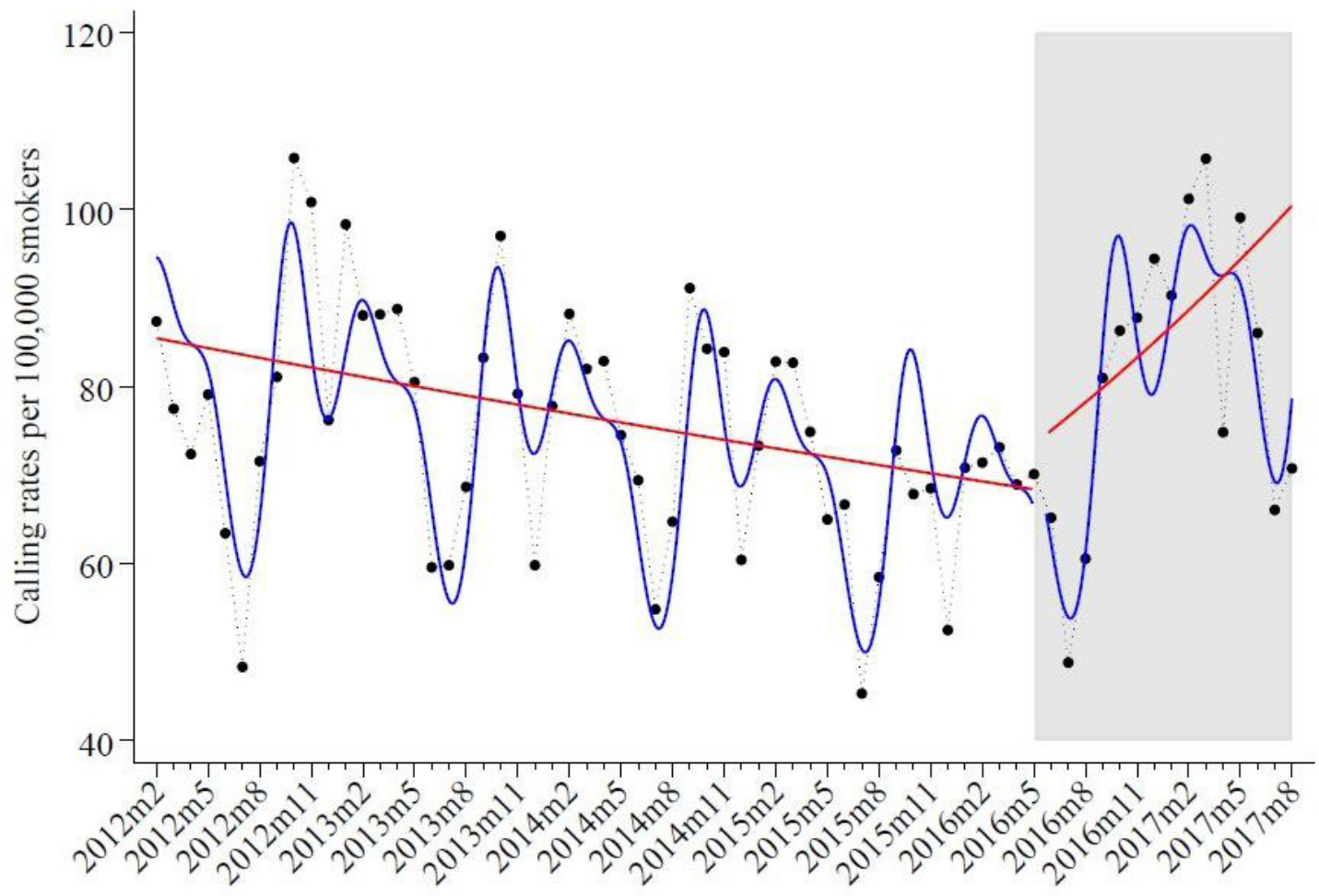

Calendar time

Figure 1 Monthly calling rate (black circle) per 100000 smokers to the Swedish smoking cessation quitline between February 2012 and August 2017. Data were fitted with Poisson regression models with calendar time modelled using degree-0 and degree-1 splines for the change in both level and trend (red solid line) and Fourier terms for the seasonality (blue line). Grey shaded area identifies the post-intervention observed period. Strings M1, M2, .., M12 indicate January, February, ..., December, respectively. The vertical axis is on the log-scale.

a simple formula to estimate the rate ratio at any postintervention month given these two parameters.

A period of 6 months was considered sufficient to capture the intervention effect and unlikely to be affected by potential time-varying confounders. The rate ratio at 6 months $\left(\mathrm{RR}_{6}\right)$ was presented to facilitate the comparison across different interventions.

To evaluate the current and the four historical interventions we defined five intervals with the criteria that one interval should contain only one intervention. Therefore, the length of the pre/post-intervention period varied depending on how close the interventions occurred over calendar time.

Analyses were performed using the tstf command in Stata (user-friendly wrapper of the tsa package in R).${ }^{67}$ Data and code are freely downloadable with the tstf package.

\section{RESULTS}

\section{Pictorial warnings and referral to quitline in May 2016}

A visual inspection of the observed calling rates per 100000 smokers (figure 1) suggests a decreasing trend in the 4 years preceding the intervention with a strong seasonal variation consisting in higher values around the end/begin (October-January) of the year and lower values in the summer time (July). During the 4 years preceding the intervention of large pictorial warnings/ text warnings and SNTQ phone number on May 2016 (figure 1), the calling rates to the SNTQ were declining at a rate of $5 \%$ per year (95\% CI 0.93 to 0.97 ). The upward shift in calling rates at the intervention month on May 2016 was negligible ( $\mathrm{RR}=1.07 ; 95 \%$ CI 0.94 to 1.23 ). The long-term downward trend, however, was reversed by the intervention ( $p$-value for change in trend $<0.001$ ). After May 2016, the calling rates to the SNTQ increased by $28 \%$ per year (95\% CI 1.09 to 1.51 ).

The data and smooth effect of the intervention comparing observed and counterfactual rate estimated with an intervention time-series model are shown in figure 2A,B, respectively. Compared with the counterfactual ones, the calling rate increased by $35 \%$ after 6 months $\left(\mathrm{RR}_{6}=1.35 ; 95 \%\right.$ CI 1.16 to 1.57$)$.

\section{Historical interventions}

Campaign on passive smoking (January 2001)

The campaign on passive smoking launched in January 2001 was associated with a higher calling rate (online supplementary table 1). Comparing to the counterfactual rates 6 month after the intervention, the calling rate significantly increased by $61 \%\left(\mathrm{RR}_{6}=1.61 ; 95 \% \mathrm{CI} 1.06\right.$ to $2.45)$. Figure $3 \mathrm{~A}$ facilitates the comparison of the observed calling rates after the campaign on passive smoking relative to the counterfactual rates had this intervention not occurred. Figure 3B shows the sharp increase in the calling rate after January 2001. 

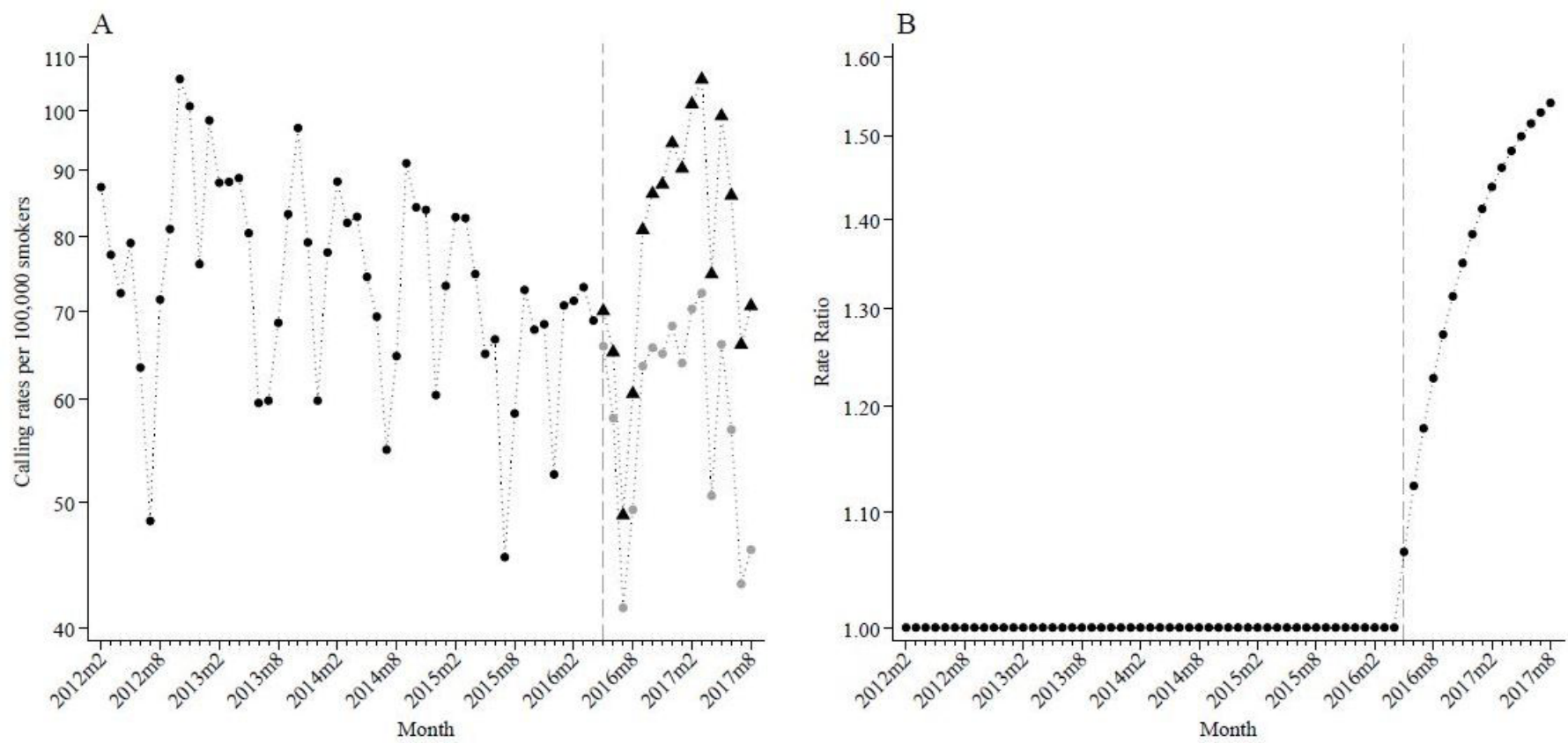

Figure 2 (A) Observed calling rates before (black circle) and after (black triangle) the intervention of implementing larger pictorial warning on the cigarette packages in May 2016. Post-intervention counterfactual calling rates, that is, predicted in the absence of intervention, are shown as grey circles. (B) Rate ratio comparing observed and counterfactual calling rates (black triangle divided by grey circle) to the Swedish National Tobacco Quitline after intervention. Data fitted with an intervention timeseries model. Strings M1, M2, ..., M12 indicate January, February, ..., December, respectively. Rates and rate ratios (vertical axis) are presented on the log-scale.

Larger text warnings on cigarette packages (September 2002)

The calling rate significantly increased after the introduction of the larger text warnings on the cigarette

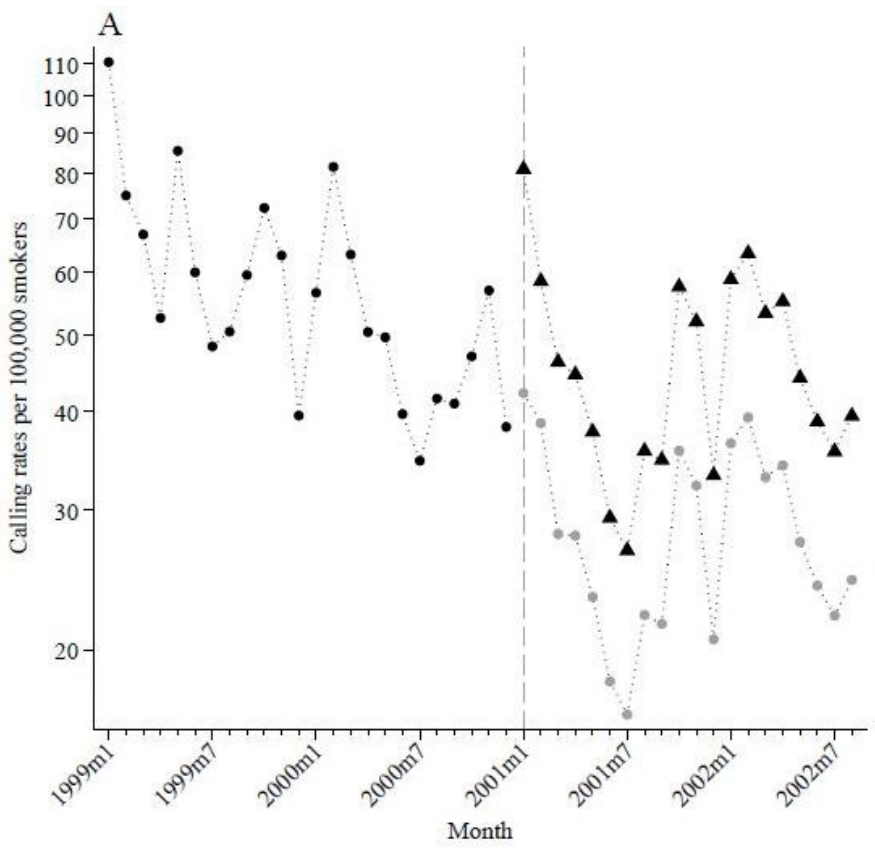

packs (online supplementary table 1 , figure $4 \mathrm{~A}$ ). The calling rate 6 months after intervention was $28 \%$ higher comparing to the counterfactual, $\mathrm{RR}_{6}=1.28 ; 95 \%$ CI 1.15

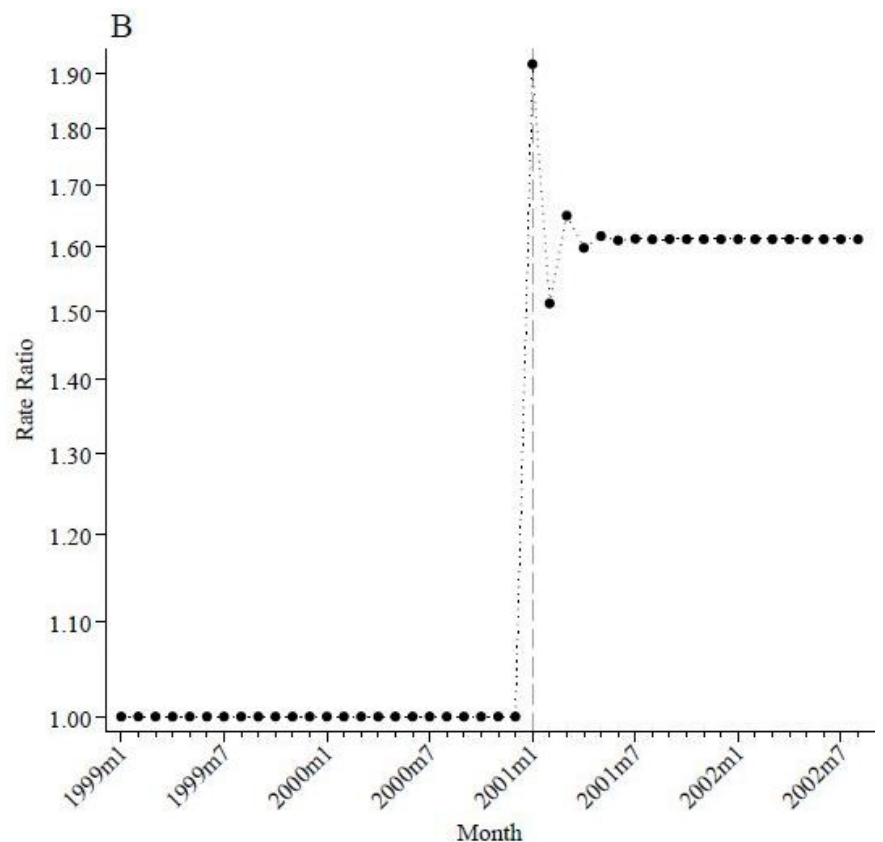

Figure 3 (A) Observed calling rates before (black circle) and after (black triangle) the intervention of implementing a campaign on passive smoking in January 2001. Post-intervention counterfactual calling rates, that is, predicted in the absence of intervention, are shown as grey circles. (B) Rate ratio comparing observed and counterfactual calling rates (black triangle divided by grey circle) to the Swedish National Tobacco Quitline after intervention. Data fitted with an intervention time-series model. Strings M1, M2, ..., M12 indicate January, February, ..., December, respectively. Rates and rate ratios (vertical axis) are presented on the log-scale. 

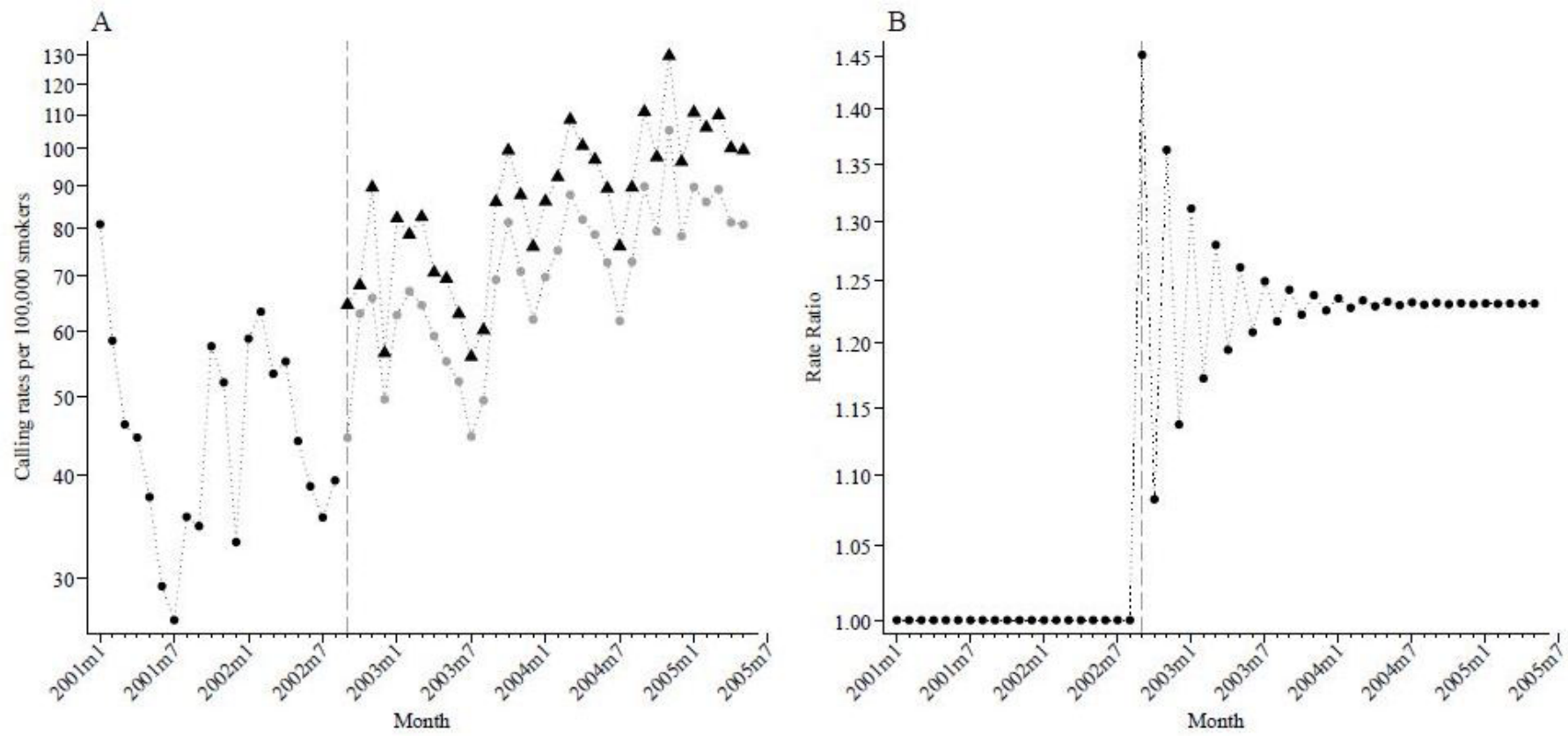

Figure 4 (A) Observed calling rates before (black circle) and after (black triangle) the intervention of larger text warnings on the cigarette packs in September 2002. Post-intervention counterfactual calling rates, that is, predicted in the absence of intervention, are shown as grey circles. (B) Rate ratio comparing observed and counterfactual calling rates (black triangle divided by grey circle) to the Swedish National Tobacco Quitline after intervention. Data fitted with an intervention time-series model. Strings M1, M2, ..., M12 indicate January, February, ..., December, respectively. Rates and rate ratios (vertical axis) are presented on the log-scale.

to 1.42). This intervention had an oscillating effect on the calling rate for several months following the intervention (figure $4 \mathrm{~A}$ ), with a stable rate ratio of about 1.2 about 12 months later (figure 4B).

\section{Banning smoking in restaurants (June 2005)}

We found a significant increased calling rate following introduction of smoking-free restaurants in June 2005 (online supplementary table 1, figure 5A). Compared with the pre-intervention months, the calling rate significantly increased by $15 \%$ after 6 months $\left(\mathrm{RR}_{6}=1.15 ; 95 \%\right.$ CI 1.01 to 1.30). Of note, banning smoking in restaurants had an oscillating effect on the calling rate for several months following the intervention (figure 5A), stabilising about 12 months later (figure 5B).

\section{Tax increase (January 2012)}

The fourth intervention consisted in a $10 \%$ tax increase on tobacco products in January 2012. The time between September 2009 and December 2014 was characterised by a relatively stable level of calling rates (online supplementary figure 1A). Data suggested that the $10 \%$ tobacco tax increase was compatible with no substantial change in the calling rate to the SNTQ (online supplementary table 1). Compared with the counterfactual rate 6 months after the intervention, the calling rate increased by $3 \%\left(\mathrm{RR}_{6}=1.03\right.$, $95 \%$ CI 0.90 to 1.19; online supplementary figure 1B).

\section{DISCUSSION}

We found differential impacts of tobacco control governmental policies implemented in Sweden during almost two decades on the propensity of smokers to call the national telephone quitline for smoking cessation. Using this propensity as impact measure, we found that a campaign on passive smoking in January 2001, larger text warnings on cigarette packs in September 2002, banning smoking from restaurants in June 2005, and finally in May 2016 the introduction of larger pictorial warnings on cigarette packages together with text referring to the telephone line for help to quit were all associated with higher calling rates in the following 6 months. Tax increment in January 2012, however, had no demonstrable impact on the calling rate.

The major strength of this study is the long time series, with information on a large number of phone calls accumulated over a long period of time (almost two decades) and collected independently of the intervention implementation. This unique source of data allowed a consistent and homogeneous assessment of the impact of diverse interventions. An additional strength regards the analytic approach, employing advanced time-series models to disentangle the effect of the intervention from the auto-correlated background and seasonal variation. The functional form specified to capture the intervention effect was sufficiently general to describe a variety of possible shapes expressed in terms of just two parameters. To facilitate the interpretation of the specified complex statistical models some effort was placed on providing both graphical comparisons of observed and predicted calling rates and closed formulas to make inference on the rate ratio associated with the intervention at any time after intervention. 

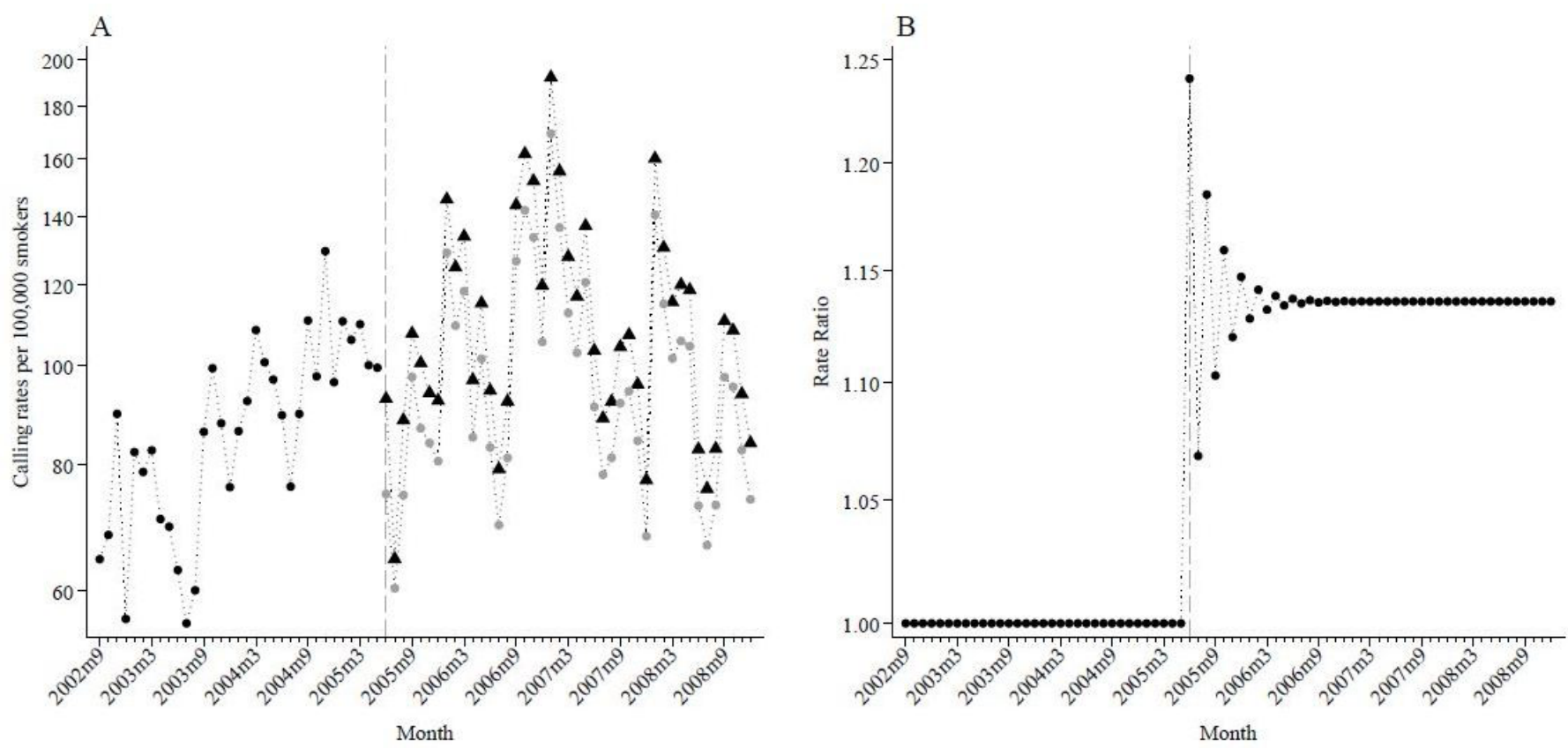

Figure 5 (A) Observed calling rates before (black circle) and after (black triangle) the intervention of banning of smoking in restaurants in June 2005. Post-intervention counterfactual calling rates, that is, predicted in the absence of intervention, are shown as grey circles. (B) Rate ratio comparing observed and counterfactual calling rates (black triangle divided by grey circle) to the Swedish National Tobacco Quitline after intervention. Data fitted with an intervention time-series model. Strings M1, M2, ..., M12 indicate January, February, ..., December, respectively. Rates and rate ratios (vertical axis) are presented on the logscale.

Limitations inherent to the collection of data on calls and the spacing of the interventions need to be mentioned. First, we could not correct the outcome variable (rate of calls) for events internal to the quitline's organisation, for instance number of staff, opening hours, marketing initiative, changes in recording and documentation system. However, no major organisation changes were recorded in the months preceding each policy launch; therefore the impact of these events if any would not coincide with the time of intervention implementation. Usually, allocation of resources and adjustments in the organisation at the quitline occur as a consequence of a recorded increase in the volume of calls, not vice versa.

Another limitation pertains the intervention components. For instance, in Sweden the last implemented policy on cigarette packages in 2016 was accompanied by an explicit reference to the SNTQ, a component that was not present during the implementation of the other policies. This should warn against the attribution of the response to the pictorial warning alone.

To evaluate multiple historical interventions, we decided to split the 19 years of follow-up into multiple intervals. Through focusing on one single intervention each time, this approach allows for measuring the intervention effects more precisely, it also offers the practical advantage of facilitating the visualisation of observed and predicted rates within a narrower interval of calendar time. On the other hand, because of the splitting, the amount of data points before each intervention will be lower, which in our case may have prevented the detection of seasonal patterns of the time-series model. Given that knowledge about the pre-intervention series defines the comparison group (or counterfactual) for the post-intervention series, limited data may result in a poor model for the pre-intervention period and a poor assessment of the intervention's effect.

The overall population of smokers in Sweden decreased from 1.3 million in 1999 to 0.7 million in 2017, that is, the prevalence of smoking in the general population nearly halved during the observation period. This may explain the variation in the absolute number of phone calls over time. This change has, however, been smooth and gradual over almost two decades. Indeed, we tried to take into account the gradual decreasing size of the smoking population directly in the definition of the outcome (calling rate rather than absolute number of calls).

Smokers taking the step to call the quitline may not be representative of all smokers wanting to quit. At its best, this behaviour may represent a precursor of a quit attempt or of relapse prevention. However, help seeking may be conceptualised as a recognition of the existence of a problem, that is, a step forward in the readiness to quit. On the other hand, studies demonstrated that smoking prevalence as assessed by surveys is also highly responsive to tobacco control measures. ${ }^{89}$

Also, inferring a causal link from temporal associations is problematic. However, several earlier observations support the plausibility of such a link. For instance, concerning the impact of the pictorial warnings it has been reported that pictorial warnings in combination 
with referral texts to a telephone quitline are judged by smokers as more effective than warnings without the referral information. ${ }^{10}$ An impact on quitline usage very similar to that observed in this study was reported in an analysis from the Netherlands after the introduction of text warnings in 2002. ${ }^{11}$ In an ecologic study comparing adult knowledge scores on smoking-related morbidity in 22 low/middle-income countries there was a clear difference in these scores among adults who noticed warning labels on cigarette packages in most countries. ${ }^{12} \mathrm{~A}$ recent study based on a pre-post survey among Italian smokers revealed an increase in knowledge on smoking-related diseases as well as in the perceived impact of pictorial warning after its implementation. ${ }^{13}$

Our study provides an appealing and cost-effective approach based on the use of routinely collected data, to corroborate these findings. Also, our study expands the evidence of policy impact by including a range of different interventions.

However, at odds with previous studies we could not detect any impact of cigarette price increase on smokers' propensity to quit. Several studies presented a temporal relation between tax increase and quitting smoking. In an Australian study ${ }^{14}$ there was an increase by $80 \%$ in selfreported quitting among smokers after a tax increase in 2010. In the USA the volume of calls at 16 state-operated quitlines increased by $59 \%$ during the 2 months preceding and following a tax increase on 1 April 2009 compared with the corresponding months 1 year earlier, ${ }^{15}$ even if no substantial difference in actual quit rates could be detected. In the US state of Montana the volume of calls to the state quitline increased by above $100 \%$ in conjunction with a tax increase in 1 January 2005, compared with equivalent periods in the year preceding and the year following that date. ${ }^{16}$

There may be several explanations for this discrepancy between our and other findings. First, different outcome measures may provide different sensitivity to policy interventions. In our study, the outcome measure is typically tailored to reflect precursors of quitting behaviour, which may not be comparable with actual quitting and even less with population smoking prevalence. Second, it is possible that price increase in Sweden affected more directly smokers whose readiness to quit was already high, thereafter less in need to call the quitline for support. Third, the entity of price increase under study may differ between countries. Finally, we cannot exclude an effect of price increase since we did observe an unexpectedly high number of phone calls in January 2012 (about fourfold higher than the average before the increase), but we adopted a conservative approach to this finding, since the number dropped back immediately during the next month.

We estimated the potential population impact of tobacco control interventions capitalising on the use of data routinely collected within national or local population-based services. This approach should be encouraged as a cost-effective way to estimate the impact of tobacco-control measures on smokers.

\section{CONCLUSIONS}

Within an overall decreasing trend of daily smoking in Sweden, we found that the recent introduction of pictorial warnings together with text warnings and referral text had a discernible positive impact on the calling rates to the smoking quitline. We were also able to detect a likely impact of earlier nationwide interventions.

Acknowledgements We gratefully acknowledge the valuable contribution of Ann Post, Sofie Jonsson and Lene Nordstrand at the smoking quitline in providing access to the data and historical information on the quitline activity and on the national policies.

Contributors $\mathrm{XZ}$ drafted the manuscript, performed the analysis and wrote the manuscript. MRG and NO conceptualised and contributed to the writing of the manuscript. AC contributed to data preparation. $\mathrm{NO}$ and $\mathrm{AC}$ contributed with advanced statistical modelling and coding. A-KD contributed in the revisions of the manuscript.

Funding This report was funded with contribution of the Public Health Agency of Sweden (SLS0 16/947).

Competing interests None declared.

Patient consent for publication Not required.

Ethics approval Regional Ethical Review Board in Stockholm approved the study.

Provenance and peer review Not commissioned; externally peer reviewed.

Data availability statement Data and code are freely available with the -tstfStata package (ssc install tstf). Dataset available at http://www.stats4life.se/data/ quitline.dta

Open access This is an open access article distributed in accordance with the Creative Commons Attribution Non Commercial (CC BY-NC 4.0) license, which permits others to distribute, remix, adapt, build upon this work non-commercially, and license their derivative works on different terms, provided the original work is properly cited, appropriate credit is given, any changes made indicated, and the use is non-commercial. See: http://creativecommons.org/licenses/by-nc/4.0/.

ORCID iD

Nicola Orsini http://orcid.org/0000-0002-2210-5634

\section{REFERENCES}

1 Zhu S-H, Lee M, Zhuang Y-L, et al. Interventions to increase smoking cessation at the population level: how much progress has been made in the last two decades? Tob Control 2012;21:110-8.

2 Stead LF, Perera R, Lancaster T. Telephone counselling for smoking cessation. Cochrane database of systematic review, 2006. Available: https://www.readcube.com/articles/10.1002/14651858.CD002850. pub2 [Accessed cited 2018 Sep 28].

3 Tomson T, Helgason AR, Gilljam H. Quitline in smoking cessation: a cost-effectiveness analysis. Int J Technol Assess Health Care 2004;20:469-74.

4 Bernal JL, Cummins S, Gasparrini A. Interrupted time series regression for the evaluation of public health interventions: a tutorial. Int J Epidemiol 2017;46:348-55.

5 GEP B, Tiao GC. Intervention analysis with applications to economic and environmental problems. Journal of the American Statistical Association 1975;70:70-9.

6 Orsini N, Zhou X-W. TSTF: Stata module to compute intervention time-series models [Internet]. Boston College Department of Economics, 2018. Available: https://ideas.repec.org/c/boc/bocode/ s458544.html [Accessed cited 2019 Jun 19].

7 Cryer JD, Chan K-S. Time series analysis: with applications in R. 2nd edition. New York: Springer, 2010: $491 \mathrm{p}$.

8 Wakefield MA, Durkin S, Spittal MJ, et al. Impact of tobacco control policies and mass media campaigns on monthly adult smoking prevalence. Am J Public Health 2008;98:1443-50.

9 Wakefield MA, Coomber K, Durkin SJ, et al. Time series analysis of the impact of tobacco control policies on smoking prevalence among Australian adults, 2001-2011. Bull World Health Organ 2014;92:413-22.

10 Hammond D, Reid JL, Driezen P, et al. Pictorial health warnings on cigarette packs in the United States: an experimental evaluation of the proposed FDA warnings. Nicotine Tob Res 2013;15:93-102. 
11 Willemsen MC, Simons C, Zeeman G. Impact of the new EU health warnings on the Dutch quit line. Tob Control 2002;11:381-2.

12 Chiosi JJ, Andes L, Asma S, et al. Warning about the harms of tobacco use in 22 countries: findings from a cross-sectional household survey. Tob Control 2016;25:393-401.

13 Mannocci A, Mipatrini D, Troiano G, et al. The impact of pictorial health warnings on tobacco products in smokers behaviours and knowledge: the first quasi-experimental field trial after the implementation of the tobacco law in Italy. Ann Ist Super Sanita 2019;55:186-94.
14 Dunlop SM, Cotter TF, Perez DA. Impact of the 2010 tobacco Tax increase in Australia on short-term smoking cessation: a continuous tracking survey. Med J Australia 2011;195:469-72.

15 Bush T, Zbikowski S, Mahoney L, et al. Us federal cigarette Tax increase and Quitline utilization in 16 states. J Environ Public Health 2009;2012.

16 Harwell TS, Lee L, Haugland C, et al. Utilization of a tobacco quit line prior to and after a tobacco Tax increase. J Public Health Manag Pract 2007;13:637-41. 